\title{
South African clinical practice guidelines: A landscape analysis
}

\author{
M Wilkinson, ${ }^{1}$ BPharm, MPH; T Wilkinson, ${ }^{1}$ BPharm, MSc (Health Economics); T Kredo, ${ }^{2}$ MB ChB, MMed (Pharm), Dip HIV Man; \\ K MacQuilkan, ${ }^{1} \mathrm{MPH}$; C Mudara, ${ }^{1}$ BSc Hons; A Winch, ${ }^{1}$ BSc Hons, MSc (Health Economics); Y Pillay, ${ }^{3}$ PhD; K J Hofman, ${ }^{1}$ MB BCh, FAAP \\ ${ }^{1}$ PRICELESS SA, School of Public Health, Faculty of Health Sciences, University of the Witwatersrand, Johannesburg, South Africa \\ ${ }^{2}$ Cochrane South Africa, South African Medical Research Council, Cape Town, South Africa \\ ${ }^{3}$ National Department of Health, Pretoria, South Africa
}

Corresponding author: KJ Hofman (karen.hofman@wits.ac.za)

\begin{abstract}
Background. South Africa (SA) is in the process of implementing National Health Insurance (NHI), which will require co-ordination of health provision across sectors and levels of care. Clinical practice guidelines (CPGs) are tools for standardising and implementing care, and are intended to influence clinical decision-making with consequences for patient outcomes, health system costs and resource use. Under NHI, CPGs will be used to guide the provision of healthcare for South Africans. It is therefore important to explore the current landscape of CPG developers and development.

Objective. To identify and describe all CPGs available in the public domain produced by SA developers for the SA context.

Methods. We conducted a cross-sectional evaluation using a two-part search process: an iterative, electronic search of grey literature and relevant websites (161 websites searched), and a systematic search for peer-reviewed literature (PubMed) after publication year 2000. CPGs were identified, and data were extracted and categorised by two independent reviewers. Any discrepancies were referred to a third reviewer. Data extracted included a description of the developer, condition, and reporting of items associated with CPG quality.

Results. A search conducted in May 2017 identified 285 CPGs published after January 2000. Of those, 171 had been developed in the past 5 years. Developers included the national and provincial departments of health (DoH), professional societies and associations, ad hoc collaborations of clinicians, and the Council for Medical Schemes. Topics varied by developer; DoH CPGs focused on high-burden conditions (HIV/AIDS, tuberculosis and malaria), and other developers focused on non-communicable diseases. A conflict of interest statement was included in $23 \%$ of CPGs developed by societies or clinicians, compared with $4 \%$ of DoH CPGs.

Conclusion. Accessing CPGs was challenging and required extensive searching. SA has many contributors to CPG development from the public and private sectors and across disciplines, but there is no formal co-ordination or prioritisation of topics for CPG development. Different versions of the CPGs were identified and key quality items were poorly reported, potentially affecting the usability and credibility of those available. There was substantial variation in CPG comprehensiveness and methodological approach. Establishing a national CPG co-ordinating unit responsible for developing standards for CPG development along with clinical quality standards, and supporting highquality CPG development, is one essential step for moving forward with NHI.
\end{abstract}

S Afr Med J 2018;108(1):23-27. DOI:10.7196/SAMJ.2018.v108i1.12825

Decisions made by healthcare professionals on the prevention and management of ill health are at the core of an effective, efficient and trusted health system. Clinical practice guidelines (CPGs) can have a substantial influence on clinical decision-making, with consequences for health outcomes, patients' access to care, health system costs and resource use.

The white paper on National Health Insurance (NHI) policy for South Africa (SA) ${ }^{[1]}$ released in June 2017 suggests that detailed treatment guidelines, based on the best available clinical and costeffectiveness evidence, will be used to guide the delivery of health services under NHI. Under NHI, standard treatment guidelines (STGs) developed by the National Department of Health (NDoH) for primary, secondary, tertiary and quaternary levels of care will play an integral role in determining access to and quality of care, and additional treatment guidelines will be used or developed where gaps in the therapeutic areas covered by the STGs are identified. In addition, the NHI policy states that 'efforts will be put into place to ensure that the general public is provided with the relevant information to support access and ensure empowerment regarding these guidelines. ${ }^{[1]}$
However, no central, accessible database of CPGs developed in SA currently exists. ${ }^{[2]}$ The CPG mapping project described in this article aimed to address this gap in knowledge of up-to-date guidelines, and to assist the $\mathrm{NDoH}$ by: ( $i$ improving the current understanding of the CPG landscape in SA; and (ii) providing a starting point for a co-ordinated CPG review and development programme under NHI. The primary objective of this project was to identify and collate all publicly available CPGs and, where available, to provide the details of the developers/commissioners of such guidance.

For the purpose of this research, and the intended NHI-focused requirements of guideline production in SA, we defined CPGs in their broadest sense as documentation that advises on the clinical management (including screening, prevention, diagnosis, treatment, rehabilitation and palliation) of individuals in a particular setting for a particular disease area/condition. The use of a more restrictive definition of CPGs, for example the Institute of Medicine's 2011 definition that includes a requirement that CPG statements/ recommendations 'are informed by a systematic review of evidence and an assessment of the benefits and harms of alternative care options, ${ }^{[3]}$ was not considered practical or appropriate, as the 
CPG landscape in SA is fragmented and not currently standardised, with limited technical skills available. ${ }^{[4]}$ As a result, the CPGs included in the database vary considerably in terms of their development methods, quality and comprehensiveness.

\section{Objective}

To identify and describe all CPGs available in the public domain, produced by SA developers for the SA context.

\section{Methods}

We conducted a cross-sectional evaluation of publicly available CPGs through an iterative, electronic search of grey literature and relevant websites, as well as a systematic search for peer-reviewed literature.

Documentation relevant to the clinical management of individuals in SA (see full definition above) produced and published after 1 January 2000 in English was included in the CPG database. Only one version or presentation of any CPG was included, and non-clinical guidelines describing ethical, legal, organisational or infrastructure factors for healthcare were excluded. Continuing medical or professional education articles and academic textbooks were also excluded.

The electronic search of grey literature and relevant websites (national and provincial departments of health, professional societies, associations, universities) was conducted between 1 September 2016 and 15 November 2016, and repeated between 22 and 25 May 2017. The list of society and association websites searched was informed by a separate Society, Association and Council Mapping Project, ${ }^{[5]}$ as well as the list provided on the Health Professions Council of South Africa's website (http://www.hpcsa. co.za/Links). In the initial search, terms including 'clinical guideline', 'treatment protocol' and 'recommendations' were used to identify websites and grey-literature sources. This was followed by a pragmatic, within-site strategy to ensure that the search was comprehensive and that sources were fully examined.

The systematic search for peer-reviewed literature was conducted in PubMed and the South African Medical Journal (SAMJ) on 18 October 2016, and updated by the first reviewer and repeated by the second reviewer on 12 May 2017. Articles published between 1 January 2000 and 12 May 2017 were identified using search terms that included 'South Africa' and variations of the following keywords: guideline; clinical management; treatment; protocol; recommend; algorithm; clinical practice guideline; decision support; managed care; diagnoses; preventive; public health; and health service. Two reviewers independently reviewed the abstracts against the prespecified inclusion and exclusion criteria, with any disagreement discussed and referred to a third reviewer if not resolved. The Preferred Reporting Items for Systematic Reviews and Meta-Analyses (PRISMA) flow chart for the literature search is presented in Fig. 1.

Two independent reviewers extracted and categorised relevant information from the CPGs, with any disagreement discussed and referred to a third reviewer if not resolved. Information extracted included a description of the developer, condition and reporting of items associated with quality CPGs (e.g. declarations of conflicts of interest, funding sources, references or evidence base, and stakeholder involvement).

\section{Results}

In total, 285 CPGs published online after the year 2000 were retrieved. Fig. 2 provides an overview of the CPGs developed and published between January 2000 and May 2017 in SA and the broad therapeutic areas they relate to. Most CPGs provide guidance on non-communicable diseases (NCDs) (46\%, 130/285), maternal, neonatal and child health (MNCH) $(21 \%, 59 / 285)$ and HIV/AIDS, tuberculosis (TB) or malaria $(12 \%, 35 / 285)$. We found that $171 / 285$ CPGs

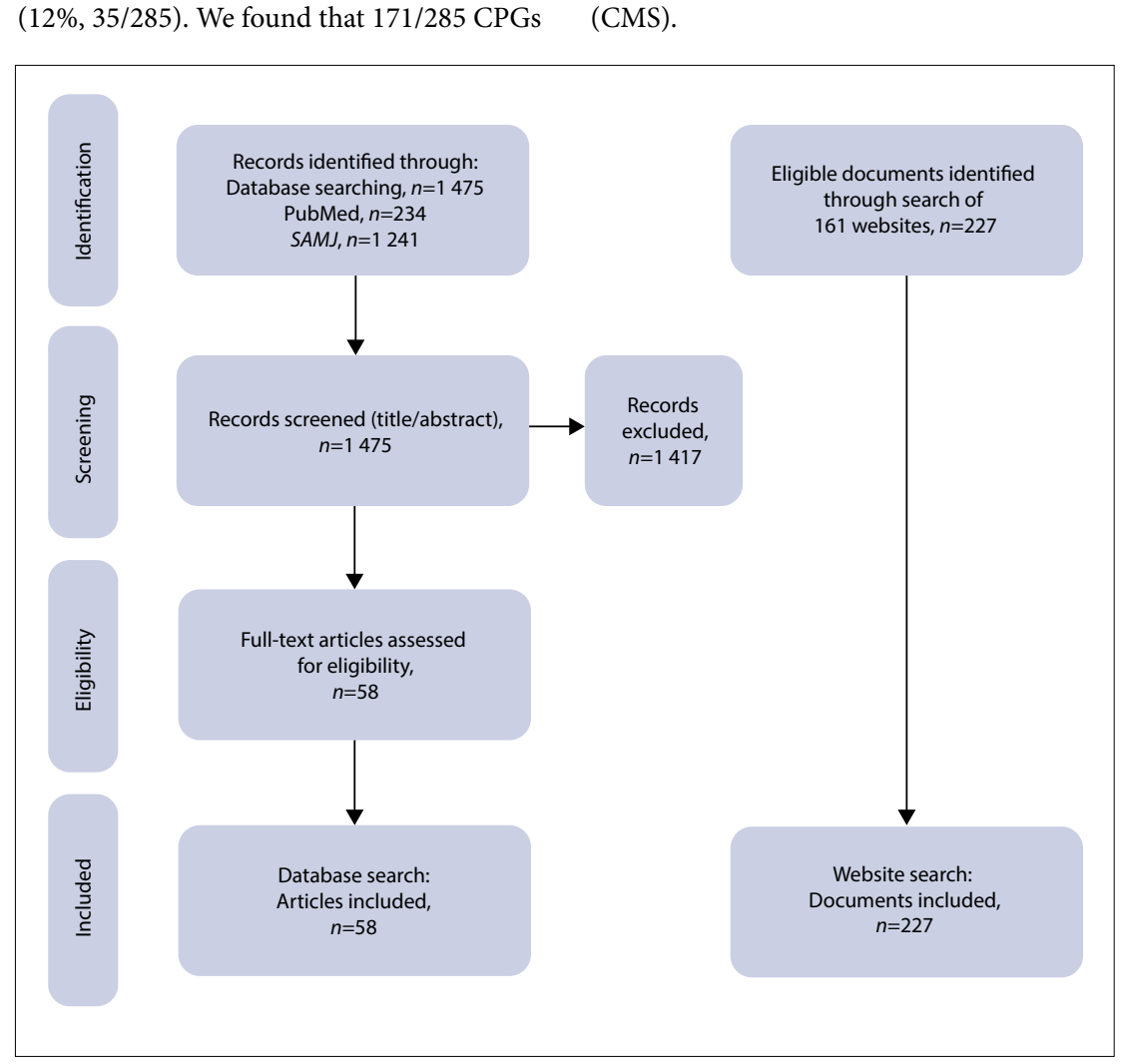

Fig. 1. PRISMA flow diagram of clinical practice guidelines (May 2017).

(60\%) were developed after 1 January 2012. The apparent increase in CPG development since 2000 (Fig. 2) could in part be explained by the fact that only the latest version of any CPG was included in the database.

CPGs were categorised based on their scope as:

- Covering multiple conditions and populations. Short guidelines/algorithms covering multiple unrelated conditions or interventions.

- Detailed. Guidelines that include the following information regarding the condition or intervention: general information, symptoms and presentation of disease, diagnosis, and management/treatment recommendations.

- Position statement. Short (usually 1 - 3 pages) recommendations or statements where the content is mainly based on the collective views of the organisation and not necessarily supported by analysis or synthesis of local evidence.

- Poster/algorithm. Algorithm or poster on the management of a particular condition or use of an intervention.

We identified five groups of CPG developers: (i) the NDoH; (ii) provincial departments of health; (iii) societies or associations; (iv) collaborations of clinicians and academics; and $(v)$ the Council for Medical Schemes (CMS) 
CPG developer types and categorisation are shown in Table 1. CPGs in each category vary in development methodology, length, target audience and scope (guidance on an individual intervention $\mathrm{v}$. management of a condition).

\section{Department of Health CPGs}

Nine $\mathrm{NDoH}$ CPGs 'covering multiple conditions and populations' were identified: (i) three STGs for SA (primary care level, and hospital level for adults and children); (ii) Tertiary and Quaternary Level Essential Medicines Recommendations, 2016; (iii) Integrated Management of Childhood Illness (IMCI), 2014; (iv) Adult Primary Care guide, 2016/2017; ( v) Newborn Care Charts, 2014 (these were developed by the Limpopo Initiative for Newborn Care, a joint initiative of the Department of Paediatrics and Child Health at the University of Limpopo and the Limpopo Department of Health, but have been categorised as an $\mathrm{NDoH}$ guideline owing to their national implementation and use); (vi) Guidelines for Neonatal Care, 2008; and (vii) the STG for common mental health conditions. Outdated versions (when newer versions of the CPGs are available) of the STGs, the IMCI and the Adult Primary Care guide (previously named Primary Care 101) were found on multiple websites, including those of the $\mathrm{NDoH}$, universities, provincial DoHs and professional societies and associations.

Most of the 'detailed' NDoH guidelines $(n=45)$ were for HIV/AIDS, TB or malaria $(28 \%, 15 / 45)$, followed by CPGs for $\mathrm{MNCH}(20 \%, 9 / 45)$, NCDs $(20 \%, 9 / 45)$ and communicable diseases and infections $(19 \%, 8 / 45)$. Some of the NDoH CPGs were adaptations of World Health Organization (WHO) guidelines, and many were developed in collaboration with, or with financial or technical support from, international development aid agencies such as the United Nations Children's Fund (UNICEF), United States Agency for International Development (USAID), United Nations Population Fund (UNFPA) and Joint United Nations Programme on HIV/AIDS (UNAIDS).

Only three of the 'detailed' CPGs by provincial DoHs were produced in the past

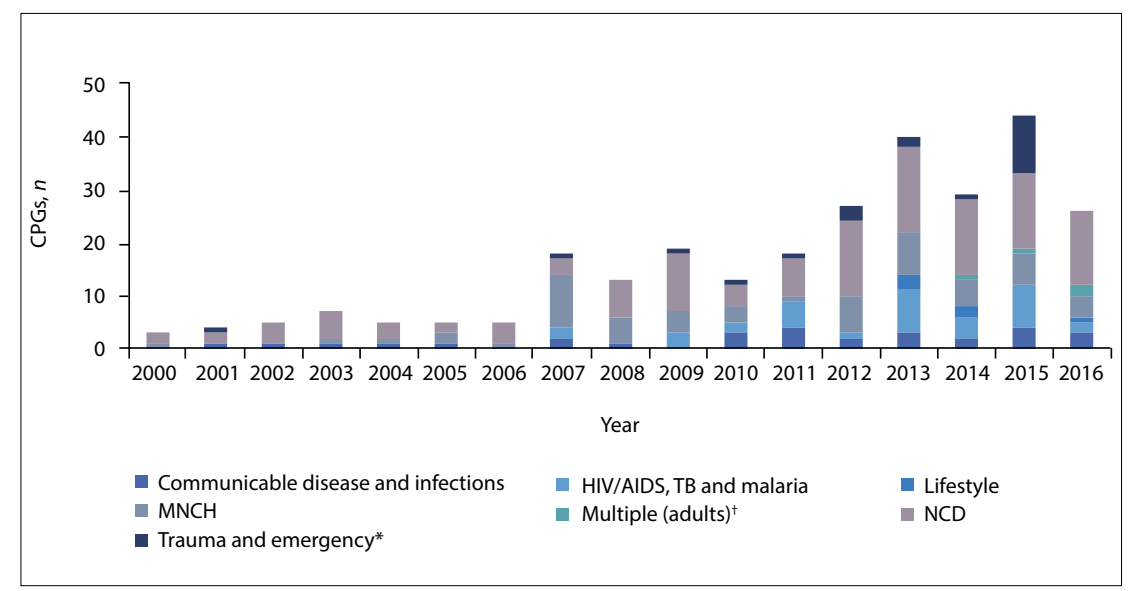

Fig. 2. CPGs produced in SA between January 2000 and May 2017 by broad therapeutic area $(\mathrm{N}=285)$. $(C P G s=$ clinical practice guidelines; $S A=$ South Africa; $T B=$ tuberculosis; $M N C H=$ maternal, neonatal, and child health; $N C D=$ non-communicable disease. ${ }^{*}$ One $C P G$ on the use of blood products in SA has been included under the 'Trauma and emergency' field; however, this document includes guidance covering more than one broad therapeutic area for both adults and children. ${ }^{\dagger} T h e$ 'Multiple (adults)' field includes four documents that consist of a package of adult-focused CPGs covering more than one broad therapeutic area. Paediatric CPGs covering more than one broad therapeutic area are included under the MNCH field.)
5 years. Two of these were developed by the KwaZulu-Natal DoH for preventing and managing malnutrition, and one by the Western Cape DoH on antimicrobial management. The KwaZulu-Natal DoH also produced protocols for management of mental health conditions, and two paediatric CPGs 'covering multiple conditions and populations' in 2007.

\section{Society or association CPGs}

A total of 156 CPGs developed by 63 societies or associations were identified, with the majority of organisations $(54 \%, 34 / 63)$ producing or contributing to more than one CPG. Some of the CPGs were adaptations of guidelines developed by professional societies outside SA.

The majority of the CPGs were 'detailed' $(67 \%, 104 / 156)$ and were mostly produced after 1 January 2012 (62\%, 96/156). Sixty percent (94/156) of the CPGs advised on the management of NCDs, with many referring to musculoskeletal (14/94), cardiovascular (13/94) and gastrointestinal (12/94) conditions.

None of the position statements identified $(n=41)$ were published in a peerreviewed journal, and they were mainly developed by five societies: the South African Spine Society $(n=12)$, the South African Vitreoretinal Society $(n=6)$, the South African Gastroenterology Society $(n=4)$, the South African Society of Cardiovascular Intervention $(n=4)$ and the South African Society of Obstetricians and Gynaecologists $(n=4)$.

Ten poster/algorithm guidelines were included in the CPG database, of which nine were produced by the Resuscitation Council of South Africa.

\section{CPGs produced by clinicians and academics}

Thirty-seven CPGs containing no formal statement linking their development to the $\mathrm{DoH}$ or a specific society or association were included as clinician/academic-produced CPGs. The majority of the CPGs were for $\mathrm{MNCH}(40 \%, 15 / 37)$, followed by NCDs

Table 1. Overview of clinical practice guidelines in South Africa by developer type

\begin{tabular}{|c|c|c|c|c|c|c|}
\hline & $\begin{array}{l}\text { National } \\
\text { Department of } \\
\text { Health, } n\end{array}$ & $\begin{array}{l}\text { Provincial } \\
\text { department of } \\
\text { health, } n \\
\end{array}$ & $\begin{array}{l}\text { Society/ } \\
\text { association, } n\end{array}$ & $\begin{array}{l}\text { Clinicians/ } \\
\text { academics, } n\end{array}$ & $\begin{array}{l}\text { Council for } \\
\text { Medical } \\
\text { Schemes, } n\end{array}$ & Total, $N$ \\
\hline Multiple conditions and populations & 9 & 3 & 1 & 0 & 1 & 14 \\
\hline Detailed & 45 & 10 & 104 & 37 & 14 & 210 \\
\hline Position statement & 2 & 1 & 41 & 0 & 0 & 44 \\
\hline Poster/algorithm & 3 & 4 & 10 & 0 & 0 & 17 \\
\hline Total & 59 & 18 & 156 & 37 & 15 & 285 \\
\hline
\end{tabular}


Table 2. Summary of findings: Items associated with good-quality CPGs

\begin{tabular}{|c|c|c|c|c|c|}
\hline & $\begin{array}{l}\text { National } \\
\text { Department of } \\
\text { Health }(n=59)\end{array}$ & $\begin{array}{l}\text { Provincial } \\
\text { department of } \\
\text { health }(n=18)\end{array}$ & $\begin{array}{l}\text { Societies / } \\
\text { associations } \\
(n=156)\end{array}$ & $\begin{array}{l}\text { Clinicians and } \\
\text { academics } \\
(n=37)\end{array}$ & $\begin{array}{l}\text { Council } \\
\text { for Medical } \\
\text { Schemes }(n=15)\end{array}$ \\
\hline Funding statement & $\begin{array}{l}\text { Funding source } \mathrm{r} \\
\text { or unclear }^{\dagger}(3 \%) \\
n=1 \text { stated } \\
\text { pharmaceutical } \\
\text { industry } \\
\text { involvement }\end{array}$ & $\begin{array}{l}\text { lot stated }{ }^{\star}(81 \%) \\
\text { None stated } \\
\text { pharmaceutical } \\
\text { industry } \\
\text { involvement }\end{array}$ & $\begin{array}{l}\text { Funding source } \\
\text { not stated }^{*} \\
(73 \%) \text { or } \\
\text { unclear }^{\dagger}(2 \%) \\
22 \% \text { declared } \\
\text { pharmaceutical } \\
\text { industry } \\
\text { involvement }\end{array}$ & $\begin{array}{l}\text { Funding not } \\
\text { stated }^{*}(59 \%) \text { or } \\
\text { unclear }^{\dagger}(5 \%) \\
32 \% \text { declared } \\
\text { pharmaceutical } \\
\text { industry } \\
\text { involvement }\end{array}$ & $\begin{array}{l}\text { Not stated } \\
(100 \%)\end{array}$ \\
\hline Conflict of interest statements, $\%$ & 0 & 17 & 21 & 30 & 0 \\
\hline References available, $\%$ & 54 & 28 & 74 & 100 & 93 \\
\hline Description of stakeholder consultation process, $\%$ & 32 & 28 & 26 & 30 & 0 \\
\hline
\end{tabular}

$(32 \%, 12 / 37)$, other communicable diseases and infections $(14 \%$, $5 / 37)$ and HIV/AIDS, TB and malaria $(8 \%, 3 / 37)$.

Most (33/37) of the CPGs were published in the SAMJ, with the rest available on journal and university websites: African Journals Online (AJOL) $(n=1)$, the Journal of Endocrinology, Metabolism and Diabetes of South Africa (JEMDSA) $(n=1)$, the University of KwaZuluNatal $(n=1)$ and the University of Cape Town $(n=1)$.

\section{Council for Medical Schemes CPGs}

Fifteen CPGs developed by the CMS were identified. These consist of one CPG 'covering multiple conditions and populations', which contains the algorithms specifying the minimum standards required (under Prescribed Minimum Benefits (PMB)) in the management of the 25 chronic conditions on the Chronic Diseases List, and 14 'detailed' Diagnosis Treatment Pair CPGs published on the CMS website as a result of the PMB Definition Project. ${ }^{[6]}$ The Diagnosis Treatment Pair CPGs all relate to cancer, cardiovascular disease and organ transplants.

\section{Key quality criteria of included CPGs}

Sixteen percent (47/285) of CPGs contained a statement regarding the authors' conflicts of interest, and 23\% (65/285) explicitly declared the funding source. The methods for stakeholder consultation as part of the CPG development process were described in 26\% (75/285) of CPGs, and 71\% (203/285) included references. A brief summary of the findings by developer type is presented in Table 2 .

Seventeen of the $59 \mathrm{NDoH}$ CPGs (29\%) stated the involvement of international development partners (e.g. WHO, UNICEF, USAID) in the CPG development process. These CPGs had a strong focus on $\mathrm{MNCH}(n=8)$ and the management of HIV/AIDS, TB and malaria $(n=6)$. Pharmaceutical industry involvement was declared or assumed (based on pharmaceutical industry advertisements directly within the CPG) in the development of 54 CPGs, of which two-thirds (36/54) were focused on NCDs.

\section{Discussion}

CPGs developed in SA vary considerably in terms of their topics, scope, development methods, funding streams and accessibility. This variability is not surprising considering the number and diversity of CPG developers, and the lack of formal co-ordination or standardisation between them with regard to CPG topic selection and prioritisation, development methodology and reporting principles.
The individual topic selection/prioritisation process followed by the developers was generally not reported, and therapeutic topics vary considerably. The majority of DoH CPGs were focused on high-burden conditions such as HIV/AIDS, TB and malaria, while CPGs from other developers were more likely to provide guidance on NCDs. One possible explanation for this variation in CPG topics is the difference in the type of conditions treated by public and private healthcare providers, and as a result the types of CPGs they need or are likely to access. The effect of the aims and objectives of funding organisations (e.g. pharmaceutical industry, international development partners) on CPG topic selection was not considered as part of this mapping project, but may warrant future research to ensure that CPG topics are prioritised and selected based on the needs of the population and the healthcare community.

CPGs identified in the public domain were often out of date (with more up-to-date versions available elsewhere) and key quality items we extracted were poorly reported, potentially impacting on the usability and credibility of those available.

Accessing CPGs was challenging, as no central database of CPGs currently exists. CPGs can be submitted to the $S A M J$ for publication, but no formal 'clinical guidelines' were published in the period between the introduction of the AGREE II assessment to the SAMJ critical appraisal process for clinical guidelines in 2014 $4^{[7]}$ and May 2017. The systematic search for CPGs in the SAMJ retrieved 87 Continuing Medical Education (CME) articles published between 2014 and May 2017 that contained the features of a CPG. However, these articles were not subject to a peer review process prior to publication $^{[8]}$ and were not included in the CPG database. Some societies, associations, departmental organisations and universities publish CPGs on their websites, but in many cases the CPGs were out of date. The Ideal Clinic programme website consistently contained up-to-date versions of most of the core DoH guidelines (https:// www.idealclinic.org.za). The Ideal Clinic programme is an $\mathrm{NDoH}$ initiative, started in 2013, with a strong focus on the use of guidelines to support its aim of 'systematically improving the quality of care provided in Primary Health Care facilities. ${ }^{[9]}$

\section{Strengths and limitations of the literature search}

A systematic approach to identifying CPGs produced in SA and extracting the relevant data was followed. Detailed inclusion and exclusion criteria were established, based on a clear scope for the literature search. Dual review and data extraction of CPGs, as well 
as a search of grey literature, were conducted to minimise potential selection bias.

Despite the comprehensive search, given the difficulty with identifying CPGs, it is probable that CPGs are missing. Non-Englishlanguage CPGs were excluded, so guidance produced in any of the other official languages in SA will not have been retrieved, and guidelines that were not dated may potentially have been missed.

Key criteria regarding funding and conflicts of interest were extracted, but a robust quality assessment of CPGs was not conducted. Two prior studies have evaluated the quality of a sample of CPGs in SA using the AGREE II checklist, and consistently found the reporting on several aspects of the methods for CPG development to be of low to moderate quality. ${ }^{[2,10]}$ Further quality appraisal on the full set of CPGs may not provide additional insight.

\section{Conclusions}

SA has a diverse CPG-developing community, but the challenges faced by clinicians in accessing up-to-date CPGs and the lack of co-ordination between developers may limit the impact of CPG developers' efforts to guide and improve the delivery of high-quality patient care.

Developing and maintaining an accessible, up-to-date CPG repository is a practical and useful first step towards improving the availability of CPGs in SA. The 285 CPGs identified through this mapping project provide a starting point for such a repository. In addition to a point of access for clinicians, this CPG database can also be used to inform the planning and determination of service benefits under NHI, and provide information for a clinical guidance gap analysis to identify topic areas where future CPG development will be most beneficial. Useful lessons can be learnt from information technology organisations such as the Open Medicines Project and Essential Medical Guidance (EMGuidance), which are already working collaboratively, developing and maintaining smartphone applications that provide access to the most up-to-date versions of $\mathrm{NDoH}$ CPGs (STGs, Tertiary and Quaternary Level Essential Medicines Recommendations, TB and HIV $)^{[1]}$ and some CPGs produced by other SA developers. ${ }^{[12]}$

Stakeholder involvement is a crucial component that needs to be incorporated in all stages of the CPG development process. The South African Medical Association (SAMA) is currently 'engaging its medical practitioner members to contribute substantively to the development of guidelines, sharing their experience and expertise in the process. ${ }^{[13]}$ This could potentially improve the credibility and acceptability of CPGs by healthcare professionals across both the private and public sectors, and ultimately result in meaningful changes in clinical practice. Clinical quality standards are useful tools that can be used to further aid and enhance CPG implementation, and evaluate their clinical impact under NHI. Patient involvement in the CPG development process should also be considered, to ensure that patients are involved and empowered in decisions affecting their health.

In addition, the findings from this CPG mapping project and the South African Guidelines Excellence (SAGE) project ${ }^{[4]}$ demonstrate the need for a national, co-ordinating CPG unit that will enable a standardised, co-ordinated and evidence-based approach to CPG development. A national co-ordinating body will be essential if CPGs are to inform patient care under NHI, with a likely impact on quality of care. Ideally it will be responsible for developing and upholding key components of CPG production, which includes robust processes for topic selection and prioritisation, development, publication/ implementation and review.

A full list of the CPGs identified is available on the PRICELESS SA website (www.priceless.ac.za).

\section{Acknowledgements. None.}

Author contributions. MW led and co-ordinated the CPG mapping project and prepared the first draft of the manuscript. YP and $\mathrm{KJH}$ formulated the research concept, determined its scope and advised on its design and presentation. TW and TK provided technical and methodological input to the research methods, data analysis and presentation of the findings. MW, KM, CM and AW contributed to the CPG review, selection and data extraction processes. All the authors reviewed the first draft of the manuscript and approved the final version to be published.

Funding. This research at PRICELESS SA was supported by the International Decision Support Initiative (IDSI) (http://www.idsihealth. org/) and funded by the Bill and Melinda Gates Foundation (IDSI 2 Contract: NICE 1082). TK's contribution was funded through the Flagships Awards Project by the South African Medical Research Council (SAMRC-RFA-IFSP-01-2013/ SAGE).

Conflicts of interest. None of the authors declares any conflicts of interest. TK conducts reviews that inform national and international guidelines, co-ordinates and facilitates training on CPG development and implementation, and is principal investigator on the SAGE project.

1. National Department of Health, South Africa. National Health Act, 2003 (Act No. 61 of 2003). National Health Insurance Policy: Towards universal health coverage. Government Gazette No. 40955, 2017.

2. Kredo T, Gerritsen A, van Heerden J, Conway S, Siegfried N. Clinical practice guidelines within the Southern African Development Community: A descriptive study of the quality of guideline development and concordance with best evidence for five priority diseases. Health Res Policy Syst 2012;10:1. https://doi.org/10.1186/1478-4505-10-1

3. Graham R, Mancher M, Wolman D, Greenfield S, Steinberg E. Clinical Practice Guidelines We Can Trust. Washington, DC: Institute of Medicine Committee on Standards for Developing Trustworthy Clinical Practice Guidelines/National Academies Press, 2011:15.

4. South African Guidelines Excellence Project. SAGE Summit Report. Cape Town: SAGE, 2016. http:// www.mrc.ac.za/cochrane/SAGESummitReport.pdf (accessed 18 July 2017).

5RICELESS SA, University of Witwatersrand. Societies and Associations Mapping Project. Johannesburg: PRICELESS SA, 2016.

6. Council for Medical Schemes. Terms of Reference: Prescribed Minimum Benefit (PMB) Definition Project. Pretoria: CMS, 2010. http://www.medicalschemes.com/files/PMB\%20Definition\%20Project/ Project. Pretoria: CMS, 2010. http://www.medica).
PMBDefProject_TOR.pdf (accessed 18 July 2017)

7. Wiseman R, Cohen K, Gray A, et al. AGREE to disagree: Critical appraisal and the publication of Wiseman R, Cohen K, Gray A, et al. AGREE to disagree: Critical appraisal and the p
practice guidelines. S Afr Med J 2014;104(5):345-346. https://doi.org/10.7196/SAMJ.8215

8. South African Medical Journal. Policies: Peer review process. http://samj.org.za/index.php/samj/ about/editorialPolicies\#peerReviewProcess (accessed 18 July 2017).

9. Ideal Clinic South Africa. Home page: What is an ideal clinic? https://www.idealclinic.org.za (accessed 18 July 2017).

10. Machingaidze S, Zani B, Abrams A, et al. Series: Clinical Epidemiology in South Africa. Paper 2 Quality and reporting standards of South African primary care clinical practice guidelines. J Clin Epidemiol 2017;83:31-36. https://doi.org/10.1016/.j.jlinepi.2016.09.015

11. Open Medicine Project. Primary Health Care app launch with the South African National Dept of Health. 2015. http://openmedicineproject.org/2015/11/primary-health-care-app-launch-with-thesouth-african-national-dept-of-health/ (accessed 7 June 2017).

12. Essential Medical Guidance. EM Guidance. https://emguidance.com (accessed 7 June 2017).

13. McGee S. Involving Medical Doctors in Clinical Practice Guideline Development. Cape Town: SAGE Matters, 2017:4. http://www.mrc.ac.za/cochrane/sage/June2017.pdf (accessed 18 July 2017). 